\title{
Million solar mass black holes at high redshift
}

\author{
Oleg Y. Gnedin \\ Institute of Astronomy, Madingley Road, Cambridge CB3 0HA, UK \\ E-mail: ognedin@ast.cam.ac.uk
}

\begin{abstract}
.
The existence of quasars at redshift $z>5$ indicates that supermassive black holes were present since the very early times. If they grew by accretion, the seeds of mass $\gtrsim 10^{5} M_{\odot}$ must have formed at $z \sim 9$. These seed black holes may result from the collapse and dissipation of primordial gas during the early stages of galaxy formation. I discuss the effects of magnetic fields on the fragmentation of cold gas clouds embedded into a hot diffuse phase and a virialized dark matter halo. The field of $10^{-4} \mathrm{G}$ ejected by supernova remnants can halt cloud break-up at $10^{4} M_{\odot}$. High star formation rates keep the clouds partially ionized, making ambipolar diffusion inefficient. The magnetically-supported clouds collapse into black holes, which later spiral via dynamical friction into a central cluster with the total mass $M_{b h} \sim 6 \times 10^{6} M_{\odot}$. As the cluster collapses, the black holes merge emitting gravitational radiation that should be detectable by LISA.
\end{abstract}

\section{Seed black holes in centers of galaxies}

Supermassive black holes have been inferred and detected in many galaxies, distant and nearby. They seem to be almost ubiquitous in large elliptical galaxies (Magorrian et al. 1998). The detection of quasars at redshift $z>5$ (Fan et al. 1999; Stern et al. 2000) implies that supermassive black holes existed when the Universe was only $t_{H} \sim 10^{9} \mathrm{yr}$ old. This amount of time is not very large on galactic scales and provides a powerful constraint on the formation of black holes.

An existing black hole can grow by accretion from the gaseous disk or disrupted stars. Accreting with a standard efficiency $\varepsilon_{M} \equiv L / \dot{M} c^{2} \sim 0.1$ and emitting at a nearly Eddington rate, $\varepsilon_{L} \equiv L / L_{\mathrm{Edd}} \sim 1$, the black hole doubles its mass in

$$
t_{\mathrm{acc}}=\frac{\varepsilon_{M}}{\varepsilon_{L}} \frac{\sigma_{T} c}{4 \pi G m_{p}} \approx 4 \times 10^{7} \mathrm{yr} .
$$

If the accretion is inefficient $\left(\varepsilon_{M} \ll 0.1\right)$, as in the ADAF (Narayan \& Yi 1994) and ADIOS (Blandford \& Begelman 1999) models, the timescale is shorter but I consider the standard scenario as an example. After about ten $e$-folding times, the black hole can grow in mass by four to five orders of magnitude. Thus, starting at $t_{H} \approx 5 \times 10^{8}$ yr (for a flat cosmological model with $\Omega_{0}=0.4$ and the Hubble constant $h=0.65$ ) a $10^{5} M_{\odot}$ black hole in the high-redshift quasar progenitor can reach the mass $\sim 10^{9} M_{\odot}$.

However, this efficient accretion mechanism requires a seed black hole in place at $z \approx 9$. At that epoch large galaxies do not yet exist. In the hierarchical formation scenario, they assemble via infall and mergers of small clumps of baryonic gas and dark matter. Even though we may never know the exact route leading to black hole formation, we can consider various scenarios and choose the most plausible. Some 
proposed scenarios involve stars, producing black holes either as an endresult of the evolution of massive stars or through a cluster of degenerate stellar remnants merging into a single object. These processes, relying on two-body relaxation of the stellar distribution, are slow and require a large number of local dynamical times, at least $10^{8}-10^{9}$ yr (Quinlan \& Shapiro 1990; Lee 1995; Lee, these proceedings). Alternatively, purely gas-dynamical scenarios seem to be more efficient (e.g., Loeb \& Rasio 1994). The collapse and fragmentation can proceed on the free-fall timescale, $\lesssim 10^{6} \mathrm{yr}$ at $r=100 \mathrm{pc}$, while the cooling time at that radius is just $5 \times 10^{3} \mathrm{yr}$.

It seems that in order to form a black hole in a gas-dynamical process, two generic constraints must be satisfied: (1) the gas must be strongly self-gravitating; and (2) star formation must be inefficient, allowing a large supply of cold gas to be converted to a black hole. This second constraint is particularly important, since star formation is a competition to the black hole formation.

In order to prevent the formation of stars, small-scale density perturbations should be effectively erased or damped. One way to achieve this is to keep the gas hot, with the thermal speed comparable to the gravitational free-fall velocity; another is to have an almost relativistic equation of state, where information is again transmitted faster than the perturbations grow. The latter criterion is satisfied in objects dominated by radiation pressure and by isotropic (well-tangled) magnetic field.

\section{Conservation of angular momentum}

To set the stage, consider the just virialized dark matter halo at $z_{\mathrm{vir}}=9$ with the mass $M_{\text {vir }} \approx 1.8 \times 10^{10} \sigma_{100}^{3}\left[10 /\left(1+z_{\text {vir }}\right)\right]^{3 / 2} M_{\odot}$ and the baryonic fraction $f_{b}=\Omega_{b} / \Omega_{0}=0.1$. Assume an isothermal density distribution of dark matter with a velocity dispersion $\sigma_{d m}=100 \sigma_{100} \mathrm{~km} \mathrm{~s}^{-1}$. As the initial density perturbation grows, the gas heats adiabatically $\left(T \propto \rho^{2 / 3}\right)$. During the mergers of pregalactic clumps, the gas is shockheated to the virial temperature and follows the dark matter profile:

$$
\rho_{g}(r)=\frac{\sigma_{d m}^{2}}{4 \pi G r^{2}} f_{b}(r)
$$

For the mean molecular weight of gas with the primordial composition, $\mu_{h}=0.6$, the corresponding virial temperature is $T_{\text {vir }}=3.6 \times 10^{5}\left(1+\mu_{b}\right) \sigma_{100}^{2} \mathrm{~K}$, where $\mu_{b}(r) \equiv \frac{1}{r} \int f_{b}(r) d r$. The initial amount of angular momentum acquired from tidal torquing at the turn-around radius of the protogalaxy is given by the dimensionless factor, which is remarkably constant in cosmological simulations:

$$
\lambda=\frac{J|E|^{1 / 2}}{G M^{5 / 2}}=0.05,
$$

where $M$ is the total mass, and $E$ is the total energy. When the gas density in the center becomes high enough that atomic cooling is fast $\left(t_{\mathrm{cool}}<t_{\mathrm{dyn}}\right)$, the gas decouples from dark matter. Cooling gas contracts until it settles into the centrifugallysupported disk with the characteristic size $R_{d} \approx \lambda R_{\mathrm{vir}}$.

The early epoch of a hierarchical galaxy assembly differs from the later times in that the infalling clumps of gas and dark matter scatter off each other, redistributing angular momentum and thus providing the effective "gravitational viscosity". Numerical simulations of mergers, with (Mihos \& Hernquist 1996) and without (Hernquist \& Mihos 1995) the effects of star formation, show that gravitational torques cause the gas lose a large fraction of its angular momentum, up to $99 \%$, in one or two local dynamical times. The gas in these simulations develops 
strong inflows and accumulates in the center. Any non-axisymmetric structures, such as a temporary bar, would speed up the process.

\section{On the possibility of a direct formation of the central object from gaseous collapse}

Consider now the extreme case when the gas loses most of its angular momentum in the subgalactic mergers. If the effective value of $\lambda$ is reduced by a factor of hundred, $\lambda_{\text {eff }}=\lambda / 5 \times 10^{-4}$, the scale-length of the gaseous disk becomes a mere $4 \sigma_{100}$ pc. Such disk is so dense that it may trap its own cooling radiation and form a supermassive star. This happens when the speed of photon diffusion, $c / \tau$, falls below the free-fall velocity. Thus, the optical depth for photon scattering needs to be at least $\tau>210 \sigma_{100}^{-1} \lambda_{\text {eff }}^{1 / 2}$ (a similar scenario has been considered by Haehnelt \& Rees 1993).

There are three possible physical processes leading to photon scattering in the primordial gas: Thomson scattering off electrons, resonant scattering of Ly $\alpha$ photons off neutral atoms, and radiation pressure due to recombination (Haehnelt 1995). Because of the very high density of the gas, the cooling time is extremely short ( $\sim 8 \mathrm{yr})$ and therefore most of the hydrogen quickly becomes neutral. The small ionization fraction $\left(x \lesssim 10^{-5}\right)$ renders inefficient both electron scattering $\left(\tau_{e} \propto x ; \tau_{e} \sim 0.03 \sigma_{100} \lambda_{\mathrm{eff}}^{-1 / 2}\right)$ and the radiation pressure due to recombination $\left(\tau_{\text {rec }} \propto x^{2} ; \tau_{\text {rec }} \sim 0.15 \sigma_{100}^{-2}\right)$. The dominant opacity comes from the Ly $\alpha$ scattering $\left(\tau_{\text {Ly } \alpha} \sim 10^{5} \sigma_{100}^{-2} \lambda_{\text {eff }}^{-3 / 2}\right)$. The high density of the gas implies a huge star formation rate, $4 \times 10^{4} M_{\odot} \mathrm{yr}^{-1}$, which provides the ionizing photons that after absorption and recombination become the Ly $\alpha$ photons. These photons are numerous enough to keep the supermassive object in radiation pressure support. (Note, however, that if $\lambda>0.01 \sigma_{100}^{-1 / 2}$ even Ly $\alpha$ scattering would not be sufficient to trap the radiation.)

The result is a Very Massive Object, which can be either a superstar or a very dense disk depending on the amount of angular momentum left. The structure of the VMO is a relativistic $\gamma=4 / 3$ polytrope dominated by radiation pressure. Such configuration is unstable to radial collapse due to the effects of general relativity ("post-newtonian instability") and implodes to a black hole, if viscous torques can remove the remaining angular momentum. The final mass of the black hole depends on how much mass the VMO can accumulate over its evolutionary timescale, $\sim 3 \times 10^{4}$ yr (Baumgarte \& Shapiro 1999). In the spherical collapse scenario, the gas accumulates in the center with a constant infall rate $\dot{M}=3.5 \sigma_{100}^{3} M_{\odot} \mathrm{yr}^{-1}$, so the resulting black hole mass can be $\gtrsim 10^{5} M_{\odot}$.

\section{Effects of the magnetic field}

If the cooling gas preserves the cosmological value of the angular momentum, $\lambda=0.05$, thermal instability in the disk leads to the two-phase media of cold clouds in pressure equilibrium with hot diffuse gas (e.g., Fall \& Rees 1985). The dynamics of cold clouds can be significantly modified in the presence of a magnetic field. A well-tangled magnetic field provides additional resistance to gravitational contraction and prevents fragmentation of clouds below a certain mass limit, the "magnetic Jeans mass" (McKee et al. 1993). The rms magnetic energy, $\Phi_{B}$, would balance the gravitational pull in 
clouds less massive than

$$
M_{\Phi}=0.12 G^{-1 / 2} \Phi_{B} \approx 700\left(\frac{B}{10^{-4} \mathrm{G}}\right)\left(\frac{L}{1 \mathrm{pc}}\right)^{2} M_{\odot},
$$

where $L$ is the size of the cloud.

There could be a primordial magnetic field in the early galaxies, generated before virialization (Gnedin et al. 2000; Kulsrud et al. 1997). But additionally, after the first significant burst of star formation, stellar winds inject metals and magnetic field lines into the interstellar medium (Rees 1994). The magnetic flux of each supernova remnant would expand in space until the remnants overlap or fade away. For example, the expanding Crab nebula is $\sim 1 \mathrm{pc}$ in size with $B_{\text {crab }} \approx 4 \times 10^{-4} \mathrm{G}$ (Hester et al. 1996). The number of SN remnants in the inner $100 \mathrm{pc}$ would be high, $N_{S N R} \sim 3 \times 10^{4}$, assuming a lifetime of the remnant of $10^{5} \mathrm{yr}$. Due to the very short mean free path in the magnetized medium, of order $10^{8} \mathrm{~cm}$, the ions and electrons behave as a fluid and the shock fronts of the SN remnants intersect but do not penetrate each other before dissipating. Therefore, the magnetic flux is determined locally by the effective scale of expansion of a single SN remnant, or $\approx 3 \mathrm{pc}$. The average magnetic field can then be as large as $4 \times 10^{-5} \mathrm{G}$.

The second generation of clouds formed within these supernova remnants would be magnetized. A large fraction of the material in the clouds is ionized and their equilibrium temperature is therefore around $T \approx 10^{4} T_{4} \mathrm{~K}$. Such clouds can be approximated as isothermal and uniform. Their structure is determined by the pressure of the hot diffuse gas. The fraction of hot gas is determined locally as the amount of gas unable to radiate away the heating by gravitational infall and by other sources. Since in the isothermal distribution, $t_{\mathrm{dyn}} \propto r$, the density of the hot media would initially be $\rho_{h}(r) \propto r^{-1}$. After the onset of star formation from cold gas, heating by stellar winds and supernova explosions adds to the energy balance and couples the amounts of available hot and cold gas:

$$
\rho_{h}(r)=1.5 \times 10^{-23} \sigma_{100}^{3 / 2} r_{100}^{-3 / 2} \Gamma^{1 / 2}\left(\frac{1+\mu_{b}}{3}\right)^{1 / 4} \mathrm{~g} \mathrm{~cm}^{-3},
$$

with $\Gamma \equiv f_{k, 0.1} f_{*, 0.1} f_{b, 0.1} \Lambda_{-23} \lambda_{0.05}^{-1} e^{-t / t_{0}} \sim 1$. Here $r_{100}$ is the galactic radius in units of $100 \mathrm{pc}, f_{k}$ is the fraction of supernova explosion energy that goes into the kinetic energy of the surrounding media, $f_{*}$ is the local fraction of cold gas that turns into stars on a dynamical timescale, and $\Lambda=10^{-23} \Lambda_{-23} \mathrm{erg} \mathrm{cm}^{-3} \mathrm{~s}^{-1}$ is the minimum of the cooling function of the primordial gas at $T \sim 10^{6} \mathrm{~K}$. The local supply of gas is consumed on a timescale $t_{0}=t_{\text {dyn }} / f_{*}$ with a star formation rate $\dot{M}_{*}=43 \sigma_{100}^{3} f_{*, 0.1} f_{b, 0.1}^{3 / 2} \lambda_{0.05}^{-3 / 2} M_{\odot} \mathrm{yr}^{-1}$. This gives the internal cloud density

$$
n(r)=1.7 \times 10^{3} \sigma_{100}^{7 / 2} r_{100}^{-3 / 2} T_{4}^{-1} \Gamma^{1 / 2}\left(\frac{1+\mu_{b}}{3}\right)^{5 / 4} \mathrm{~cm}^{-3},
$$

and the Jeans mass

$$
M_{J} \approx 3.4 \times 10^{5} \sigma_{100}^{7 / 4} r_{100}^{3 / 4} T_{4}^{2} \Gamma^{-1 / 4}\left(\frac{1+\mu_{b}}{3}\right)^{-5 / 8} M_{\odot} .
$$

If the cloud cools enough that the value of the Jeans mass falls below its mass, $M_{c l}$, the cloud starts contracting and fragmenting into smaller, denser subclouds which cool further. This process can go on until $M_{c l} \sim M_{\Phi}$, at which point the magnetic pressure will resist fragmentation. Since both the gravitational and magnetic energies 
scale with the cloud size as $L^{-1}$, the cloud will remain in marginal equilibrium at any value of $L$. Thus, any increase in the external pressure will cause uniform cloud collapse. Unless the magnetic flux can be effectively dissipated, the inevitable outcome of such contraction is the formation of a compact supermassive object.

In Galactic molecular clouds, the magnetic flux is removed by ambipolar diffusion of ions through neutral atoms. The diffusion timescale is

$$
t_{\mathrm{ad}} \approx 9 \times 10^{11} x\left(\frac{L}{1 \mathrm{pc}}\right)^{2}\left(\frac{n}{10^{3} \mathrm{~cm}^{-3}}\right)^{2}\left(\frac{B}{10^{-4} \mathrm{G}}\right)^{-2} \mathrm{yr},
$$

where $L$ is the scale of the magnetic field variation (Draine, Roberge \& Dalgarno 1983). The efficiency of ambipolar diffusion depends on the ionization fraction, $x$. The cores of molecular clouds are shielded from external ionizing radiation $\left(x \lesssim 10^{-7}\right)$ and the magnetic field decays to allow further fragmentation and star formation. In the primordial clouds, the X-rays from first star clusters should be able to penetrate the whole cloud. The X-ray luminosity at $1 \mathrm{keV}$ resulting from a solar mass of star formation per year can be empirically estimated to be $\sim 3 \times 10^{40} \mathrm{erg} \mathrm{s}^{-1}$ ( $\mathrm{Oh}$ 2001). This leads to a significant ionization fraction, $x_{c} \sim 2 \times 10^{-3}$, and a long ambipolar diffusion time, $t_{\mathrm{ad}} \sim 2 \times 10^{9} \mathrm{yr}$. Therefore, the clouds with mass $M_{\Phi}$ would remain magnetically supported. Also, a frozen uniform magnetic field is important for removing cloud's angular momentum via "magnetic braking" on roughly a dynamical time of the ambient medium (McKee et al. 1993).

The dynamics of cold clouds is regulated by two competing effects, condensation from the hot phase and destruction in predominantly inelastic collisions. Thermal instability of the virialized gas leads to the formation of clouds on a cooling timescale. The binding energy of these clouds is relatively small; the escape velocity $\sim 2\left(M_{3} / L_{p c}\right)^{1 / 2} \mathrm{~km} \mathrm{~s}^{-1}$. The clouds in the disk move with a much higher velocity, $v_{\text {rel }} \gtrsim 10 \mathrm{~km} \mathrm{~s}^{-1}$, and therefore cloud collisions would quickly disband them. The time between collisions depends on the number of clouds within the inner $100 \mathrm{pc}$. Assuming that the clouds develop a scale-free mass distribution $d N_{c l} / d M \propto M^{-2}$ with $M_{\min } \approx M_{\Phi}$ and $M_{\max } \approx M_{J}$, the smallest clouds would survive long enough for magnetic braking to operate if $M_{\Phi} \approx 3 \times 10^{4} r_{100}^{-3 / 4} \sigma_{100}^{-13 / 4} M_{\odot}$.

Once the magnetically-supported cloud loses enough angular momentum, external pressure perturbations would cause an unlimited uniform collapse to a black hole through an intermediate stage of a supermassive star. During the radiation-supported VMO phase, strong winds and outflows may reduce the final mass by roughly a half, although the exact value is uncertain. The VMOs close to the center would spiral in via dynamical friction off the gas and stars. Allowing time $t_{\mathrm{df}}=3 \times 10^{8} \mathrm{yr}$ for the dynamical friction with the circular velocity $\sim 170 \sigma_{100} \mathrm{~km} \mathrm{~s}^{-1}$, the radius of the swept-up region is about $r_{\mathrm{df}}=30 \mathrm{pc}$ (Binney \& Tremaine 1987) and the critical cloud mass $M_{\Phi}=1.5 \times 10^{4} \sigma_{100}^{-7} M_{\odot}$. This requires the magnetic field $B \sim 4 \times 10^{-4} \mathrm{G}$ on the scale of the cloud, $L_{\Phi} \sim 2 \mathrm{pc}$. The number of $M_{\Phi}$ clouds within the central $30 \mathrm{pc}$ is $\sim 780$, which including the mass loss of the VMO results in the cluster of black holes with the total mass $\sim 6 \times 10^{6} M_{\odot}$. The cluster keeps contracting until it dominates the gravity in the center and the dynamical friction becomes inefficient. The size of the black hole cluster at that point is $R_{c l}=0.9 \sigma_{100}^{-2} \lambda_{0.05} \mathrm{pc}$.

Notice the strong dependence of the black hole mass on the value of the magnetic field and the circular velocity of the halo, $M_{b h} \propto M_{\Phi} \propto B^{3} \sigma^{-7}$. Small galaxies forming first would have a higher mass of the magnetized clouds and also require a weaker magnetic field to support them. 


\section{Gravitational radiation from merging black holes}

The subsequent evolution of the cluster of black holes is relatively well understood. A self-similar collapse occurs (Cohn 1980) until binaries form via gravitational radiation (Quinlan \& Shapiro 1987), their eqs. (41)-(44). Because of the small number of objects in the cluster the initial relaxation time is short, $t_{\mathrm{rel}}=6.5 \times 10^{4} \mathrm{yr}$, but the initial binary capture time is long, $t_{\text {cap }}=3.3 \times 10^{10}$ yr. Binary formation becomes effective when the cluster core collapses to $\sim 0.02 R_{c l}$ on the collisionless timescale $t_{c c}=330 t_{\mathrm{rel}} \sim 2 \times 10^{7}$ yr. Since this time is shorter than the local Hubble time $\left(t_{H}=5 \times 10^{8} \mathrm{yr}\right)$, most of the black holes undergo hierarchical binary mergers through gravitational wave radiation.

The merger of a large number of black holes would lead to a significant amount of gravitational radiation from the galactic center. The comoving density of halos with $M_{\text {vir }}=2 \times 10^{10} M_{\odot}$ at $z=9$ is $\sim 7 \times 10^{-3} \mathrm{Mpc}^{-3}$. A coalescence of two $10^{4} M_{\odot}$ black holes would lead to a gravitational wave amplitude $h \sim 0.1 r_{g} / R_{H} \sim 3 \times 10^{-20} M_{4}$, where $r_{g}$ is the gravitational radius of the black hole and $R_{H}$ is the Hubble distance. The maximum frequency of gravitational radiation is $\nu_{\max } \lesssim 0.1 c / r_{g} \sim 1 M_{4}^{-1} \mathrm{~Hz}$. The mergers would proceed hierarchically, doubling the mass in each event. Thus, the last merger of the $3 \times 10^{6} M_{\odot}$ black holes gives the maximum amplitude $h \sim 10^{-17}$.

Such gravitational signal should be detectable by LISA. The early black holes should originate in the highest density peaks and therefore be highly clustered. They should appear in the same direction as the high-redshift quasars.

I would like to thank M. Rees, M. Begelman, J. P. Ostriker, and E. Zweibel for critical discussions, and the LISA conference organizers for hospitality.

\section{References}

Baumgarte T. W., Shapiro S. L., 1999, ApJ, 526, 941

Binney J., Tremaine S., 1987, Galactic Dynamics (Princeton: Princeton University Press)

Blandford R. D., Begelman M. C., 1999, MNRAS, 303, L1

Cohn H., 1980, ApJ, 242, 765

Draine B. T., Roberge W. G., Dalgarno A., 1983, ApJ, 264, 485

Fall S. M., Rees M. J., 1985, ApJ, 298, 18

Fan X. et al. (SDSS collaboration), 1999, AJ, 118, 1

Gnedin N. Y., Ferrara A., Zweibel E. G., 2000, ApJ, 539, 505

Haehnelt M. G., 1995, MNRAS, 273, 249

Haehnelt M. G., Rees M. J., 1993, MNRAS, 263, 168

Hernquist L., Mihos J. C., 1995, ApJ, 448, 41

Hester, J. J. et al., 1996, ApJ, 456, 225

Kulsrud R. M., Cen R., Ostriker J. P., Ryu D., 1997, ApJ, 480, 481

Lee H. M., 1995, MNRAS, 272, 605

Loeb A., Rasio F. A., 1994, ApJ, 432, 52

Magorrian J. et al., 1998, AJ, 115, 2285

McKee C. F., Zweibel E. G., Goodman A. A., Heiles C., 1993, in Protostars and Planets III, ed. E. H. Levy \& J. I. Lunine (Tucson: Univ. Arizona Press), 327

Mihos J. C., Hernquist L., 1996, ApJ, 464, 641

Narayan R., Yi I., 1994, ApJ, 428, L13

Oh S. P., 2001, ApJ, 553, 499

Quinlan G. D., Shapiro S. L., 1987, ApJ, 321, 199

Quinlan G. D., Shapiro S. L., 1990, ApJ, 356, 483

Rees M. J., 1994, in Cosmical Magnetism (Kluwer), ed. D. Lynden-Bell, 155

Stern D. et al., 2000, ApJ, 533, L75 